\title{
Researches on Stress Concentration and Fatigue Damage for Ferromagnetic Specimen by Permeability Testing Technology
}

\author{
Ren Shangkun ", Ren Xianzhi, Yang Meifang \\ Key Laboratory of Nondestructive Testing of Ministry of Education, Nanchang Hangkong University, Nanchang, China
}

Email address:

renshangkun@yeah.net (Ren Shangkun)

${ }^{*}$ Corresponding author

\section{To cite this article:}

Ren Shangkun, Ren Xianzhi, Yang Meifang. Researches on Stress Concentration and Fatigue Damage for Ferromagnetic Specimen by Permeability Testing Technology. International Journal of Mechanical Engineering and Applications. Vol. 5, No. 4, 2017 , pp. $203-207$. doi: 10.11648/j.ijmea.20170504.13

Received: May 7, 2017; Accepted: May 22, 2017; Published: July 10, 2017

\begin{abstract}
The permeability testing technology are new testing and evaluating method, which based on the electromagnetic induction principle, can realize the test and evaluate high sensitively of stress concentration and fatigue damage for ferromagnetic specimen. Taking Q235 and 45 steel for examples, the relationships of the detection signal and the tensile stress, residual stress, fatigue damage are studied from experiments. The results indicate that permeability testing technology can be used effectively to measure the stress state of specimen and the maximum stress suffered before. According to the residual stress signal after suffer tensile, the maximum stress to be suffered before can be determined with high sensitively. It shows that detection sensitivity of the fatigue damage is less than that of stress concentration. The detection sensitivity of fatigue damage for Q235 steel is greater than that of steel 45 steel. The results indicate that the permeability testing technology has a broad application prospect.
\end{abstract}

Keywords: Non-Destructive Testing, Permeability Detection, Residual Stress, Fatigue Damage

\section{Introduction}

In modern industry, a large number of steel components are used in the aerospace, railway, power, pressure vessels and other industries. Steel components may lead to stress corrosion, function aging and fatigue fracture easily in their using due to undergo stress concentration and fatigue damage and loading [1]. Major accidents and disasters may take place due to role of internal media and external environment which ultimately will bring disaster to the country and the people [2]. Therefore, in the non-destructive testing for the component, detecting the stress concentration and fatigue damage of critical areas quickly, accurately and conveniently is of significance in the prevention of the fracture failure of components and the occurrence of a major disaster [3-4]. Magnetic permeability testing technology is a kind of evaluation method for detecting stress concentration and fatigue damage [5-7]. This method has very high detecting sensitivity [8-12]. It not only can detect the degree of stress concentration and the fatigue damage status for ferromagnetic material specimen, but can also detect martensite-austenite transformation, mechanics toughness-brittleness transition, dislocation defect density, cementite and ferrite transformation, etc [13-14].

\section{Detection Principle}

The structure of detection probe is shown in Figure 1, which comprises a detection coil and an excitation coil and was wound on a tubular plastic frame, and it is suitable for detecting rod-shaped ferromagnetic specimen. When being tested, the test specimen was put into the tube of the sensor, the excitation coil was connected with an Alternating voltage, and a closed magnetic circuit was formed by the ferromagnetic specimen and the external environment. The stress concentration leads to the change of magnetic 
permeability, which and then leads to the change of the magnetic resistance and flux for the closed magnetic circuit. By detecting the inducing signal and permeability changed for the specimen, the status of stress distribution and degree of fatigue damage were measured.

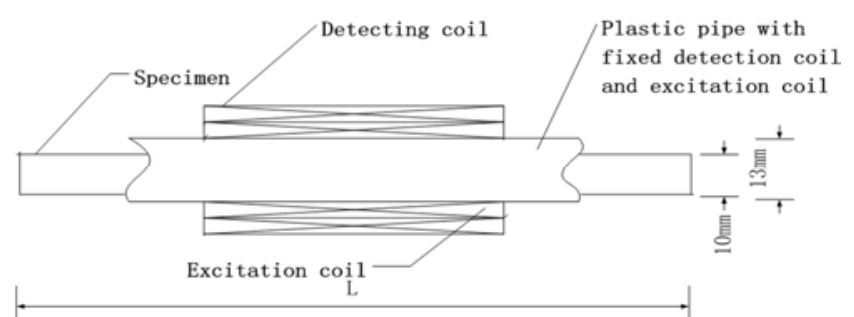

Figure 1. Sensor structure diagram.

A constant voltage source was selected as the source of excitation. According to the principle of electromagnetic induction, the output signal of the detection coil changes with the change of magnetic flux density on tube center region. By the induction coil voltage value can reflect changes in the permeability of ferromagnetic materials. The method realizes the detection of stress concentration and fatigue damage of ferromagnetic specimens.

Based on magnetic circuit loop theorem and magnetic Ohm's Law: $i=\Phi \mathrm{R}_{\mathrm{m}}, i$ express current density of the solenoid, $R_{m}$ express the magnetic resistance of full magnetic circuit, and $\Phi$ express the magnetic flux. Therefore, $\Phi=i / \mathrm{R}_{\mathrm{m}}, \mathrm{R}_{\mathrm{m}}$ consist of $R_{m}$ out and $R_{m}$ in, which expressed the magnetic resistance of air part and magnetic resistance of specimens respectively in the magnetic circuit. $R_{m}$ out magnetic resistance of air part is constant. Therefore, $\Phi=i / \mathrm{R}_{\mathrm{m}}=i /\left(\mathrm{R}_{\mathrm{m}}\right.$ out $+R_{m}$ in $), \Phi==i /\left[R_{m}\right.$ out $\left.+\mathrm{L} /(\mu \mathrm{S})\right], \mu$ express permeability of specimen, $\mathrm{L}$ is the length of specimen, $\mathrm{S}$ express the average equivalent cross-sectional area. According to Maxwell second equation:

$\oint E \cdot d l=-\int_{s} \frac{\partial B}{\partial t} \cdot d s$, flux density $\mathrm{B}=\Phi / \mathrm{S}$

output signal can be obtained:

$$
\begin{aligned}
& u_{\text {out }}= N_{2} \oint E \cdot d l=-\frac{N_{2}}{S} \int \frac{\partial \Phi}{\partial t} \cdot d s \\
& u_{\text {out }}=-\frac{N_{2}}{S} \int \frac{\partial \Phi}{\partial t} \cdot d s=-N_{2} \frac{1}{R_{m_{\text {out }}}+L /(\mu S)} \frac{d i}{d t}
\end{aligned}
$$

$\mathrm{N}_{2}$ express number of turns of the detecting coil. formula (1) indicates that when the permeability $\mu$ of the specimen to be changed, the output signal can change Correspondingly.

Negative express the phase relation of current and voltage. Due to stress concentration, fatigue damage can affect the permeability, so the structure status of stress concentration fatigue damage for specimen can be judgment and evaluate by output signal voltage amplitude.

To determine the specimen, $\mu$ is determined, the measuring value of $\mathrm{di} / \mathrm{dt}$ is constant. Formula (1) can be expressed $u_{\text {out }}=a \mu /(\mu+\mathrm{b})$, a and $\mathrm{b}$ are constant. Based on test data, the constant of $\mathrm{a}$ and $\mathrm{b}$ can be obtained.

\section{Test Equipment and Test Methods}

At room temperature, the electronic tensile test equipment of DW-100 was used for experiment, which was conducted at constant velocity tensile loading, specimen of Q235 steel and 45 steel. The maximum tensile force can not exceed $100 \mathrm{kN}$, and loading rate of $2 \mathrm{~mm} / \mathrm{min}$.

The electronic fatigue test equipment (INSTRON 8801) was employed at room temperature. Related parameters of fatigue test are as follows: stress ratio of 0.1 , sine wave, loading frequency of $15 \mathrm{~Hz}$, static load of $15.4 \mathrm{kN}$, dynamic load of $12.6 \mathrm{kN}$. Test specimens of steel rod, diameter of $10 \mathrm{~mm}$ and length of $250 \mathrm{~mm}$, were measured.

\section{Experiment and Analysis}

The experiment of static load stretching and unload were performed for Q235 steel and 45 steel. Static load stretching can determine the relation of detection signal and the tensile stress. However, unload can determine the relationship between the detection signal and the residual stress. The residual stress is caused by the loading stress, which is the stress state of the specimen after the loading stress to be canceled.

\subsection{Yield Strength and Tensile Strength}

Yield strength and tensile strength of the specimen can be obtained by measuring the stress-strain curve. Figure 2 represent the stress-strain curve of the specimens. Figure 2 (a) shows that the yield strength of Q235 specimen is $266 \mathrm{MPa}$ and the tensile strength is $416 \mathrm{MPa}$. Figure 2 (b) shows that the yield strength of 45 steel specimen is $382 \mathrm{MPa}$ and the tensile strength is $603 \mathrm{MPa}$.

\subsection{Relationship Between the Rate of Change of Permeability and Tensile Stress}

The sensor was fixed on the specimen surface and the specimen was fixed on stretcher. With stress increasing constantly, the relationship between the rate of change of permeability and the tensile stress is shown in Figure 3.

Figure 3 shows the curves of the rate of change of permeability with the tensile stress. Figure 3 (a) and Figure 3 (b) respectively show the relationship between the rate of change of permeability of Q235 and 45\# steel specimens with the tensile stress. Figure 3 (a) shows that the rate of change of permeability is slightly increased in the early stage of the tension and is reduced slowly when the stress was near the yield strength $(248 \mathrm{MPa})$. When tensile stress is at the range of $248 \mathrm{MPa}$ to $388 \mathrm{MPa}$, the permeability is reduced rapidly. When stress is near tensile strength, the rate of change of permeability can reach $-7.5 \%$. Figure 3 (b) shows when tensile stress is at the range of $75 \mathrm{MPa}$, the permeability is slightly increased. When tensile stress is at the range of $75 \mathrm{MPa}$ to $382 \mathrm{MPa}$, the permeability decreases slowly with tensile stress increasing. At the range of $382 \mathrm{MPa}$ to $603 \mathrm{MPa}$, the permeability decreases quickly with the increasing in stress. When stress is near tensile strength, the rate of change of 
permeability can reach $-7.5 \%$. In the initial stage of tensile stress, the change of permeability is mainly caused by the action of the specimen and the drawing machine. It shows that

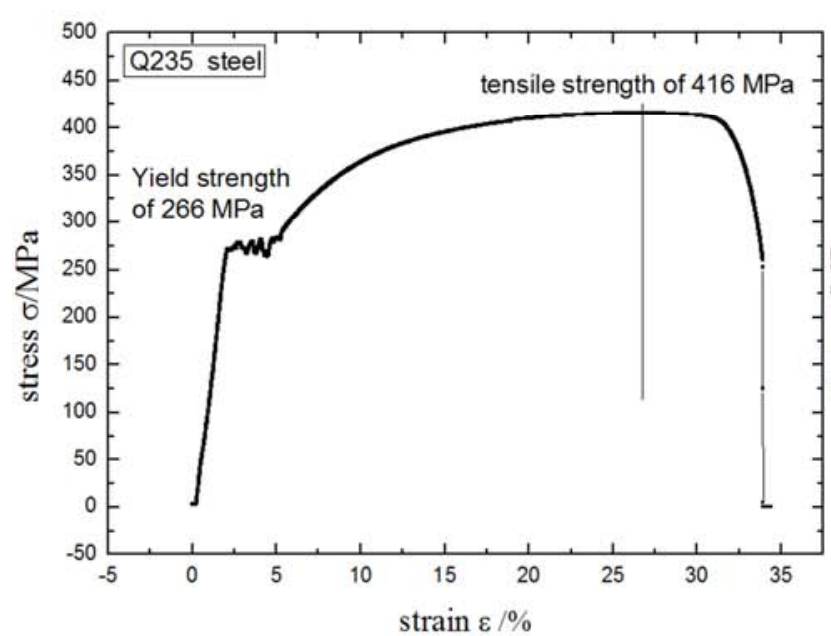

(a) stress-strain curve for Q235 steel specimen the stress state of the rod material specimen can be effectively measured based on the permeability measurement technique.

Figure 2. Stress -Strain curves for specimens.

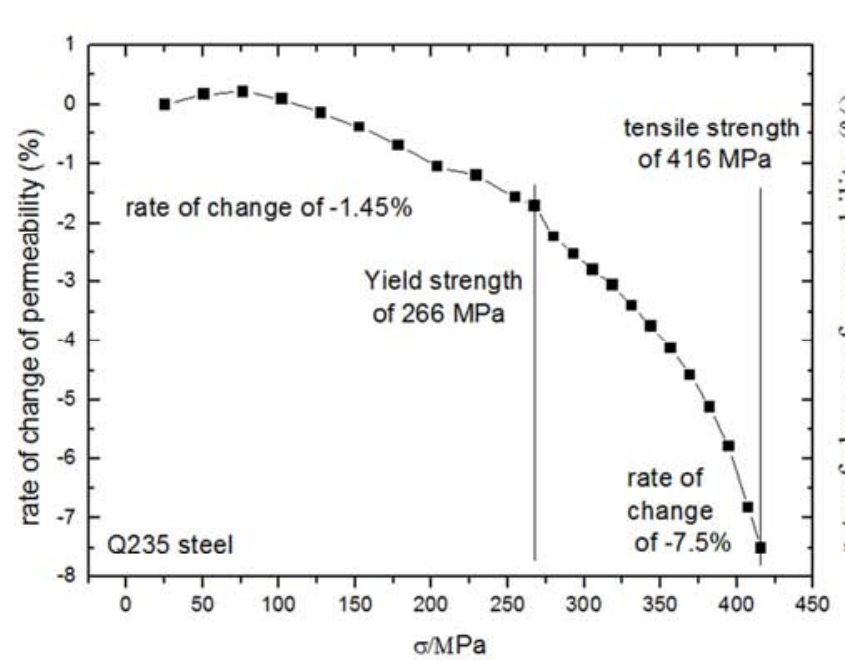

(a) rate of change of permeability versus tension stress for Q235 steel

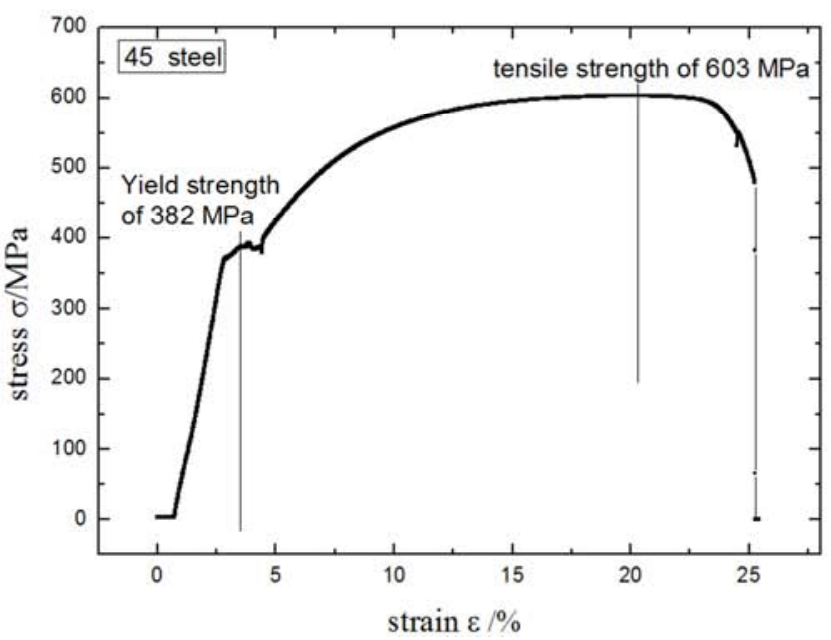

(b) stress-strain curve for 45 steel specimen

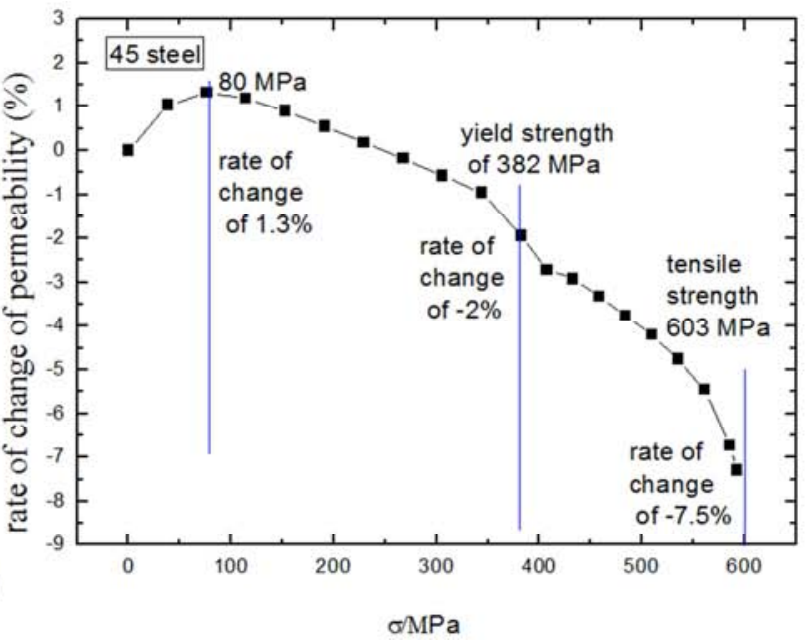

(b) rate of change of permeability versus tension stress for 45 steel

Figure 3. Tensile stress dependence of rate of change of permeability.

Figure 3 shows that the detection signal related to stress state of the specimen. The change in detection signal can reflect the change of permeability, the stress state of the specimen can be judged by measuring permeability. The absolute changing amount of signal is $0.33 \mathrm{~V}$ for Q235 steel and the relative rate of change of signal could reach $7.5 \%$. For 45 steel, the absolute signal variation is $0.3 \mathrm{~V}$ and the relative rate of change of signal could reach $7.5 \%$.

\subsection{The Influence of Tension Residual Stress on Permeability}

Figure 4 express the relationship between the rate of change of permeability and the residual stress of the tension which is the stress state of the specimen after the loading stress to be canceled, and the effects of stress and residual stress on permeability have been compared and analyzed. Figure 5 (a) and Figure 5 (b) indicate the influencing features of stress and residual stress on permeability respectively for Q235 and 45 steel.

Figure 4 shows that the detection signal of the residual stress is more sensitive than that of tension stress. The rate of change of permeability is more suitable for testing residual stress of the specimen. In the residual stress test, the changing absolute value of the signal of Q235 steel is $0.8 \mathrm{~V}$ and the relative change of signal can reach $20 \%$. The absolute signal change is $1 \mathrm{~V}$ and the relative signal change can reach $26 \%$ for 45 steel. Figure 4 (a) and figure 4 (b) show that the stress load status of 45 steel and Q235 steel are very different from that of stress unload state. The detection signal varies greatly with the increase of the residual stress, which is obviously larger than the that of detection signal caused by stress. It is shown that the change of permeability caused by residual stress is larger 
than that caused by tension. The detection technique has higher testing sensitivity of measuring residual stress than that of measuring tension. For Q235 steel and 45 steel, when the tensile stress is less than the yield strength, at the elastic

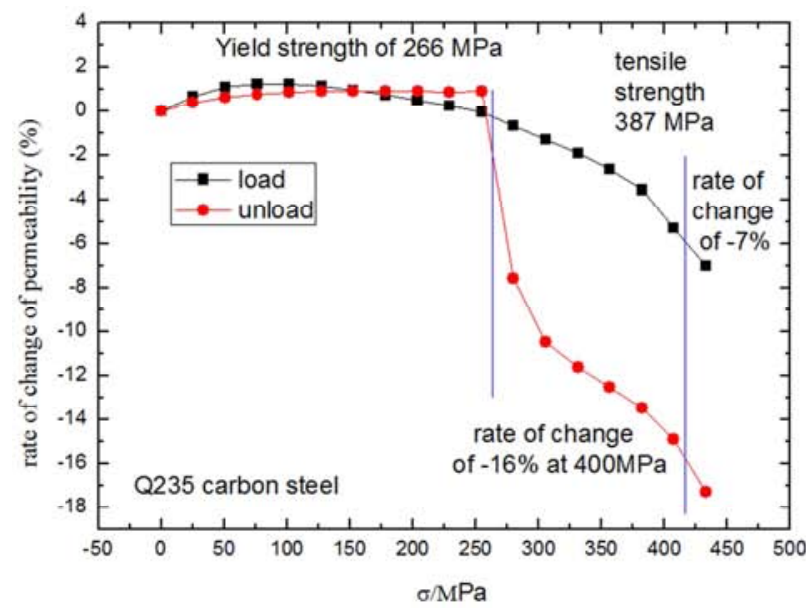

(a) Q235 steel specimen deformation range, residual stress of unloading and the detection signal near remained constant. When the tensile stress is greater than the yield strength, the detection signal decreases obviously with the increasing of residual stress.

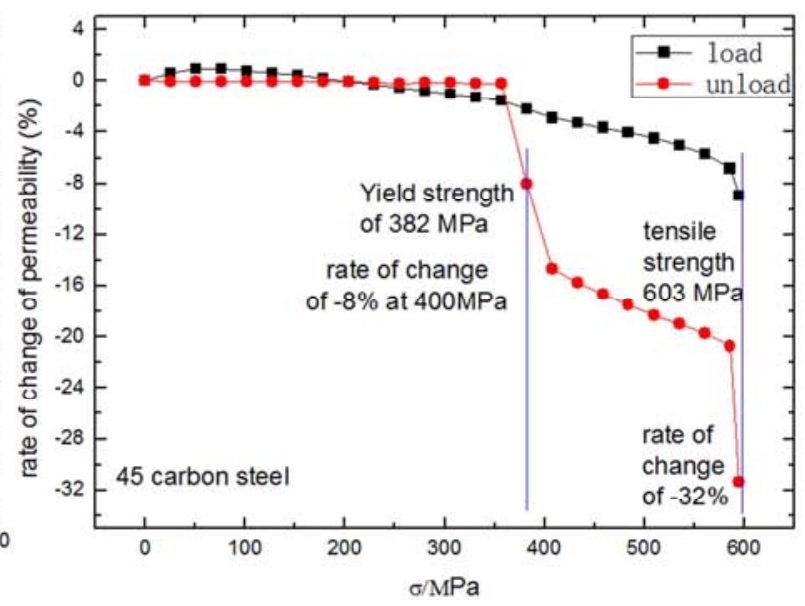

(b) 45 steel specimen

Figure 4. Rate of change of permeability versus tension stress and residual stress.

Figure 4 (a) shows that the detection signal exist a dramatic change at the $250 \mathrm{MPa}$ near of yield point, which be caused by sharp increase of residual stress for elastic deformation of the material. When the tensile stress was greater than $300 \mathrm{MPa}$, the performance of the detection signal expression a slow decline until the fracture was caused by tensile stress. The necking stage, the sample begins to undergo uneven plastic deformation, with atomic slip blocking the accumulation of defects, the residual stress increasing sharply. 45 steel and

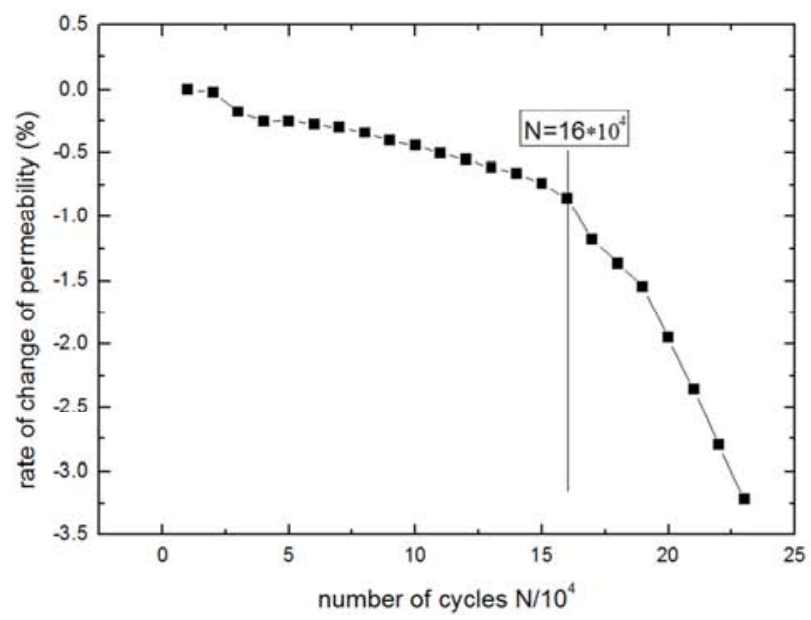

(a) Q235 steel specimen
Q235 both have similar laws.

\subsection{Effect of Fatigue Damage on the Detection Signals}

The fatigue damage experiment is similar that of static load tensile. The data were measured and record by subjecting the specimen to some hundred thousand cycles until the specimen was pulled off.

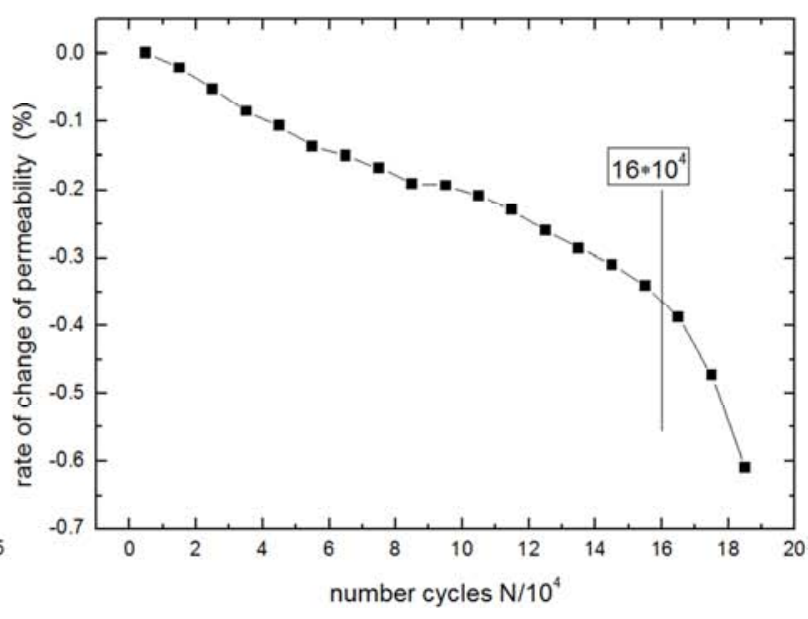

(b) 45 steel specimen

Figure 5. Rate of change of permeability as a function of number of cycle.

Figure 5 shows the relation between the detection signal and the number of fatigue cycles. Figure 5 (a) and Figure 5 (b) indicate experimental laws of Q235 and 45 steel respectively. Figure.5 (a) indicates the relation of rate of change of permeability versus number of cycles, with the maximum tensile strength of $356 \mathrm{MPa}$ greater than the yield strength of $266 \mathrm{MPa}$, which reacting the law of the low cycle fatigue. Figure 5 (b) express the rule of the high cycle fatigue of $45 \#$, with the maximum tensile strength of $356 \mathrm{MPa}$ lower than the yield strength of $382 \mathrm{MPa}$.

As it can be seen from Figure 5, the detection signal sensitivity of fatigue damage detection is lower than the stress concentration detection. Throughout the test of fatigue damage process, the relative variation of signal for Q235 was $0.13 \mathrm{~V}$, and the relative change is $3.3 \%$. The relative variation of signal for 45 steel only was $0.035 \mathrm{~V}$, and the relative change 
is $0.6 \%$. The results showed that the detection sensitivity of fatigue damage for Q235 is higher than that of 45 steel.

Figure 5 (a) and experimental data show that the detection signal slowly decreases before the 160 thousand cycles, the signal change is $33 \mathrm{mV}$, the variation accounted for $25 \%$ of the total variation. The detection signal variation of $25 \%$ is caused by $75 \%$ of cycles of fatigue. When the number of cycles of fatigue ranged from 160 thousand times to 220 thousand times, the detection signal was sharply decreased, the signal variation reached $97 \mathrm{mV}$. It can be observed that the detection technique is feasible for testing of fatigue damage and assessment of residual life for Q235.

Figure 5 (b) and experimental data show that for the fatigue before the 180 thousand cycles, the detection signal decreases slowly, that the signal has a change of $10 \mathrm{mV}$, and that the detection signal variation of $29 \%$ is caused by $95 \%$ of cycles of fatigue. When the number of times of fatigue ranges from 180 thousand times to190 thousand times, the detection signal decreases sharply, and the signal variation can reach $25 \mathrm{mV}$. The detection signal variation of $75 \%$ is caused by $71 \%$ of cycles of fatigue.

\section{Conclusion}

Permeability detection technique is a foreseeing detection method for ferromagnetic specimen with high accuracy. The testing method can predict stress concentration condition, degree of fatigue damage and aging degeneration features in some areas based on the changes of magnetic induction intensity in the probe. In the closed magnetic circuit, the microscopic structural change of specimen, must result in the changes of permeability for components. Through the experimental study for Q235 and 45\# steel specimens, the following conclusions can be obtained:

Based on the permeability measurement technique, the stress concentration condition and degree of fatigue damage of the rod ferromagnetic specimen can be effectively measured.

(2) Based on the remaining residual stress, the maximum stress can be determined that the component had bored and supported the stress condition before.

(3) It shows that the detection of residual stress has higher detection sensitivity than the measured stress, and the detection of stress concentration has a higher detection sensitivity than that of fatigue damage.

(4) When testing stress concentration, if the stress is less than the yield strength, the change of the detection signal with the stress is smaller. When the stress is greater than the yield strength, the detection signal with the stress changes obviously.

(5) When the residual stress is measured caused by tensile, if the stress is less than the yield strength, the detection signal almost unchanged with the changing of stress, and when the stress is greater than the yield strength, the changing of the detection signal is sharply with the stress.

Testing sensitivity of stress concentration and fatigue damage are related with the kind of steel and the carbon content of the steel.

\section{Acknowledgements}

This research was supported by the Natural Sciences Fund (51261023).

\section{References}

[1] Yang E, Li L. M, Chen X, Magnetic field aberration induced by cycle stress [J]. Journal of magnetism and magnetic materials, 2007, 312 (1): 72-77.

[2] S. K Ren, et al. Studies on stress-magnetism coupling effect for 35 steel components [J]. Insight: Non-Destructive Testing and Condition Monitoring, 2010, 52 (6): 305-309.

[3] S. K. Ren, et al. Influences of environmental magnetic field on stress magnetism effect for 20 steel ferromagneticspecimen [J]. Insight: Non-Destructive Testing and Condition Monitoring, 2009, 51 (12): 672-675.

[4] Dong Lihong, Xu Binshi, Dong Shiyun, et al. Variation of stress-induced magnetism signals during tensile testing of ferromagnetic steels [J]. NDT\&E International, 2008, 41: 184-149.

[5] Li Xiaomeng, Lu Kefei, Li Getian. Magnetic memory effect in static tension of steel of Q235 [J]. Physical Testing, 2013, 31 (5): 10-13.

[6] Yu Runqiao, Xu Changying. Based on the metal magnetic memory drill nondestructive evaluation techniques [J]. Journal of iron and Steel Research, 2011, 23 (11): 59-62.

[7] Duan Zhenxia, Ren Taikun, Xi Xiaowen. magnetization reversal characteristics of $40 \mathrm{Cr}$ steel stress magnetization process [J]. Journal of iron and Steel Research, 2016 (1).

[8] I. Tomas, O Stupakov, J Kadlecova, O. Perevertov Magnetic adaptive testing-low magnetization, high sensitivity assessment of material modifications. Journal of Magnetism and Magnetic Materials, 2006, 304 (2): 168-171.

[9] Wan Z, Liao J, Wang Y, et al. Research on metal tubing pit corrosion monitoring based on potential-array method [J]. Chinese Journal of Scientific Instrument, 2011, 32 (1): 19-25.

[10] Liu J, Feng J. Research on leak fault intelligent detection method for fluid pipeline based on fuzzy classification [J]. Chinese Journal of Scientific Instrument, 2011, 32 (1): 26-32.

[11] Gao W, $\mathrm{Hu} \mathrm{Y}, \mathrm{Mu} \mathrm{X}$, et al. Real-time detection and segmentation of submerged-arc welding defects in X-ray radiography images $[\mathrm{J}]$. Chinese Journal of Scientific Instrument, 2011, 32 (6): 1215-1224.

[12] G. Vertesy, T. Uchimoto, I. Tomas, T Takagi. Nondestructive characterization of ductile cast iron by magnetic adaptive testing. Journal of Magnetism and Magnetic Materials, 2010, 322 (20): 3117-3121

[13] I. Tomas. Magnetic Adaptive Testing of Non-magnetic Properties of Ferromagnetic Materials. Czechoslovak Journal of Physics, 2004, 54, (4): 23-26

[14] G. Vertesy, I. Tomas, I. Meszaros. Non-destructive indication of plastic deformation of cold-rolled stainless steel by magnetic adaptive testing. Journal of Magnetism and Magnetic Materials, 2007, 310 (1): 76-82. 